\title{
Resposta de sementes de amendoim a diferentes doses de radiação gama $\left({ }^{60} \mathrm{Co}\right)^{1}$
}

\author{
Tatiana da S. Santos ${ }^{2}$, Francisco de A. C. Almeida ${ }^{3}$, Taís de M. F. Suassuna ${ }^{4}$, Wirton M. Coutinho 4 \& Pollyne B. A. Almeida ${ }^{5}$
}

\begin{abstract}
RESUMO
A radiação gama, proveniente da fonte de ${ }^{60} \mathrm{Co}$, é bastante utilizada para esterilização, visando a prevenção da decomposição e a toxidez de origem microbiana em diversos produtos. 0 grau de radiossensibilidade de um embrião vegetal depende da espécie, do estágio de seu desenvolvimento durante a radiação, da dose empregada e do critério usado para medir o efeito biológico, sendo comumente utilizado o teste de germinação. 0 bjetivou-se, com este trabalho, estudar a sensibilidade do amendoim a radiação gama e seus efeitos na germinação, no vigor e na micoflora das sementes da cultivar BRS Havana, irradiadas com uma fonte de ${ }^{60} \mathrm{C} 0$, tipo gammacell, com taxa de dosagem de $12,5 \mathrm{kGy} \mathrm{h}^{-1}$. As doses testadas em $\mathrm{kGy}$ foram as seguintes: $0 ; 0,5 ; 1,0 ; 1,5 ; 2,0 ; 2,5 ; 3,0 ; 6,0 ; 9,0 ; 12,0 ; 15,0 ; 18,0 ; 21,0$ e 24,0. Com os resultados obtidos, concluiu-se que as doses acima de 3,0 kGy prejudicaram a viabilidade das sementes e as doses acima de $12 \mathrm{kG}$ y comprometeram totalmente 0 vigor e a germinação das sementes de amendoim. A radiação a partir da dose 2,0 kG y eliminou os fungos Aspergillus flavus e Aspergillus niger e, a partir da dose 3,0 kGy, eliminou o Aspergillus glaucus. 0 Penicilium spp. permaneceu em mais de $30 \%$ das sementes em todos os tratamentos com radiação, não sendo eliminado até a dose de $24 \mathrm{kGy}$.
\end{abstract}

Palavras-chave: vigor, germinação, análise sanitária

\section{Response of peanut seeds to different levels of gamma radiation $\left({ }^{60} \mathrm{Co}\right)$}

\begin{abstract}
The gamma radiation from ${ }^{60} \mathrm{Co}$ source is widely used for sterilization aiming at preventing decomposition and toxicity from microbes in several products. The degree of radiosensitvity of a plant embryo depends on the species, the development stage during radiation, doses used and the criteria used to measure the biological effect, the germination test, being commonly used.This work aimed to study the peanut sensitivity to gamma radiation and its effects in the germination, in the vigour and seeds microflora of cultivar BRS Havana, irradiated with ${ }^{60} \mathrm{Co}$ source, type gammacell with rate of dosage of $12.5 \mathrm{kGy} \mathrm{h}^{-1}$. The tested doses were the following: 0, 0.5, 1.0, 1.5, 2.0, 2.5, 3.0, 6.0, 9.0, 12.0, 15.0, 18.0, 21.0 and $24.0 \mathrm{kGy}$. With the obtained results, it was concluded that doses over $3.0 \mathrm{kG}$ y damaged the seeds viability, doses over $12.0 \mathrm{kG}$ totally compromised the vigour and the peanut seed germination. Radiation over the dose of $2.0 \mathrm{kG}$ y eliminated the fungus Aspergillus flavus and Aspergillus niger, and over the dose of $3.0 \mathrm{kG}$ y eliminated the Aspergillus glaucus. The Penicilium spp. remained in more than $30 \%$ of seeds in all treatments with radiation, not being eliminated until doses of $24.0 \mathrm{kG}$.
\end{abstract}

Key words: vigour, germination, health analysis

\footnotetext{
1 Parte da Dissertação de Mestrado do primeiro autor ao Curso de Pós-Graduação em Engenharia Agrícola da UFCG

${ }^{2}$ Mestre em Engenharia Agrícola. Fone: (83) 3335-2245. Email: tatysilvasantos@gmail.com

3 U AEA/UFCG. Av Aprígio Veloso 882, Bodocongó, CEP 58109-970 Campina Grande, PB. Fone: (83) 3310-1550. Email: almeida@deag.ufcg.edu.br ${ }_{4}^{4}$ Pesquisador Embrapa Algodão, Av. 0 svaldo Cruz 1143, Centenário, CEP 58.428-095 Campina Grande, PB. Fone: (83) 3182-4300 Email: tais@cnpa.embrapa.br; wirton@enpa.embrapa.br

${ }^{5} \mathrm{G}$ raduanda em Biologia, Bolsista de iniciação cientifica do CNPq. e-mail:pollynecaroca@hotmail.com
} 


\section{INTRODUÇÃO}

O amendoim (Arachis hypogaea L.) é uma das oleagionosas mais importantes e a quarta em produção, depois de soja, algodão e canola; participa com 10\% da produção mundial de óleo comestível, com uma produção em torno de 23,5 milhões de toneladas/ano; os principais produtores são Índia, China e Estados Unidos. Atualmente, a produção nacional é de aproximadamente 305,0 mil toneladas/ano e o estado de São Paulo se situa como principal produtor (CONAB, 2008).

A importância econômica do amendoim está relacionada ao fato de suas sementes possuírem sabor agradável e serem ricas em óleo (cerca de 50\%) e proteína (22 a 30\%). Além disso, contêm carboidratos, sais minerais e vitaminas, constituindo-se em um alimento altamente energético (585 calorias/100g de sementes). O sabor agradável torna o amendoim um produto destinado também ao consumo "in natura" como aperitivo, salgados, torrado e preparado de diversas formas; na indústria de doces, como grãos inteiros com diversas coberturas ou grãos moídos na forma de paçoca ou substituindo a castanha de caju em cobertura de sorvetes. Além do consumo "in natura", os grãos também podem ser utilizados para extração do óleo empregado diretamente na alimentação humana, na indústria de conservas como, por exemplo, de enlatado e em produtos medicinais.

As sementes de amendoim possuem um tegumento delicado, razão por que seu manuseio durante o processamento, o armazenamento e o transporte, lhes causa sérias injúrias, provocando redução na qualidade fisiológica e na capacidade de armazenamento, além de favorecer a entrada de patógenos (Sanders et al., 1981).

A utilização da radiação gama nos alimentos é feita para os mais diversos fins, tais como: tempo de vida útil, desinfecção de agentes inibidores de brotamento (germinação) e inativação de organismos que os degradam, entre outros.

Os efeitos da radiação são influenciados por diversos fatores, dentre os quais se citam a dose de radiação (Santos, 1993), as condições de armazenamento depois da irradiação (Brock \& Franklin, 1996), o teor de água do material a ser submetido a irradiação (Conger \& Carabia,1972) e nível de oxigênio (Bump et al., 1982).

A radiação gama não só pode afetar a germinação e o vigor mas também ser utilizada no controle de micro-organismos. Temse utilizado, em alguns estudos, esta técnica, para verificar a influência sobre a dose de radiação na eliminação da microbiótica fúngica.

Tendo em vista a influência desses fatores, a falta de trabalhos a respeito da viabilidade das sementes irradiadas com ${ }^{60} \mathrm{Co}$, principalmente nas sementes de amendoim, estudou-se o efeito de diferentes doses de irradiação gama e seu efeito na germinação, vigor e micoflora de sementes de amendoim, cultivar BRS Havana.

\section{MATERIAL E MÉTODOS}

\section{Local do experimento e origem das sementes}

Este trabalho foi desenvolvido na Unidade Acadêmica de Engenharia Agrícola (UAEA) da Universidade Federal de Campina Grande (UFCG). A irradiação das amostras de semen- tes foi feita no Departamento de Energia Nuclear (DEN) da Universidade Federal de Pernambuco (UFPE), em Recife, Pernambuco. Os ensaios para avaliação da microflora, vigor, germinação e determinação da umidade foram conduzidos nos Laboratórios de Fitopatologia e Química da Embrapa Algodão (CNPA), em Campina Grande, Paraíba.

As sementes utilizadas nesses experimentos foram da cultivar BRS Havana e produzidas na cidade de Mogeiro, na Paraíba, na safra 2006/2007. Utilizaram-se todas as recomendações para o cultivo, como adubação, conforme análise de solo e controle de pragas e de doenças. As sementes foram armazenadas em casca até a sua utilização, no município de Patos, na Paraíba.

\section{Tratamento das sementes com fontes de radiação ${ }^{60} \mathrm{Co}$}

As irradiações das sementes de amendoim, cultivar BRS Havana, para controle da micoflora, foram feitas em um irradiador, que emite raios gama a partir de uma fonte de ${ }^{60} \mathrm{Co}$. As amostras de $300 \mathrm{~g}$ de sementes, com teor de umidade de $6,8 \%$ correspondentes a cada parcela experimental, foram acondicionadas em sacos de polietileno transparente antes de serem colocadas no irradiador, para evitar contaminação das sementes após a irradiação por exposição ao ambiente. As doses de radiação gama utilizadas foram $0 ; 0,5 ; 1 ; 1,5 ; 2 ; 2,5 ; 3 ; 6 ; 9 ; 12 ; 15 ; 18 ; 21 \mathrm{e} 24$ $\mathrm{kGy}$. Depois da radiação as sementes foram transportadas em suas embalagens lacradas, para o Laboratório de Fitopatologia da Embrapa Algodão, onde se realizaram os testes de vigor, germinação e determinação da micoflora. Antes e após o tratamento com radiação determinou-se a umidade das sementes de forma não destrutiva por meio de um equipamento de Ressonância Magnética Nuclear ${ }^{1} \mathrm{H}$ de baixo campo (RMN).

\section{Teste de vigor e germinação}

Utilizaram-se, para avaliar a germinação, cinco repetições de 50 sementes para cada tratamento, cujo substrato foi em papel germitest, com duas folhas de papel na base e uma na cobertura, que foram umedecidas com água destilada esterilizada, enroladas e, posteriormente, colocadas em recipientes plásticos em incubadora do tipo BOD, sob temperatura constante de $28 \pm 2^{\circ} \mathrm{C}$, de acordo com as regras para análise de sementes (Brasil, 1992).

Determinou-se a percentagem de germinação somandose as sementes que germinaram na primeira contagem, realizada no quinto dia após a semeadura, com as sementes que germinaram na segunda contagem, efetuada no décimo dia. As contagens e a apreciação das plântulas obedeceram às prescriçõos oficiais para análise de sementes (Brasil, 1992).

O teste de vigor foi realizado simultaneamente com o teste de germinação, por meio do teste indireto da primeira contagem da germinação, conforme descrito por Vieira \& Carvalho (1994).

\section{Avaliação da micoflora}

$\mathrm{Na}$ avaliação da micoflora associada às sementes irradiadas, empregou-se o método do papel de filtro umedecido (blot- 
ter test); nesse teste se utilizaram 50 sementes (cinco repetições de 10 unidades) para cada tratamento. As sementes, em número de dez, foram distribuídas no interior de placas de Petri de $15 \mathrm{~cm}$ de diâmetro, sobre uma camada constituída de três folhas de papel de filtro previamente esterilizadas e umedecidas com água destilada e esterilizada e incubadas em BOD a temperatura de $24 \pm 2{ }^{\circ} \mathrm{C}$ e fotoperíodo de doze horas de luz, durante sete dias. $\mathrm{Na}$ identificação e contagem dos fungos se examinaram as sementes, individualmente, ao microscópio estereoscópico, após sete dias de incubação; em alguns casos, a identificação foi confirmada pela visualização das estruturas morfológicas dos fungos ao microscópio óptico.

\section{Delineamento experimental e análise estatística}

Os experimentos de germinação e vigor foram dispostos em delineamento inteiramente casualizado, com quatro repetições e sete tratamentos, correspondentes às seguintes doses de radiação, em kGy: $\mathrm{T}_{1}=0,0 ; \mathrm{T}_{2}=0,5 ; \mathrm{T}_{3}=1,5 ; \mathrm{T}_{4}=3,0$; $\mathrm{T}_{5}=6,0 ; \mathrm{T}_{6}=12,0 ; \mathrm{T}_{7}=24,0$.

O programa estatístico utilizado na análise dos dados foi o SAS (SAS/STAT, 2000). Os dados obtidos foram submetidos ao teste $\mathrm{F}$ e, por se tratar de uma variável quantitativa (doses), utilizou-se análise de regressão, de acordo com recomendações de Santos et al. (2007).

Testaram-se, na avaliação da micoflora, 14 de doses de irradiação $\left(\mathrm{T}_{1}=0,0 ; \mathrm{T}_{2}=0,5 ; \mathrm{T}_{3}=1,0 ; \mathrm{T}_{4}=1,5 ; \mathrm{T}_{5}=2,0\right.$; $\mathrm{T}_{6}=2,5 ; \mathrm{T}_{7}=3,0 ; \mathrm{T}_{8}=6,0 ; \mathrm{T}_{9}=9,0 ; \mathrm{T}_{10}=12,0 ; \mathrm{T}_{11}=$ 15,$0 ; \mathrm{T}_{12}=18,0 ; \mathrm{T}_{13}=21,0 ; \mathrm{T}_{14}=24 \mathrm{kGy}$ ) dispostas em delineamento inteiramente casualizado com quatro repetições. Os resultados obtidos foram analisados conforme estatística descritiva da porcentagem de contaminação de cada fungo identificado, em função das doses de radiação.

\section{RESULTADOS E DISCUSS ÃO}

\section{Vigor e germinação}

A umidade das sementes foi determinada antes e após o processo de irradiação; antes de serem irradiadas, a umidade média das sementes foi de $6,8 \%$ e após o processo, de $6,9 \%$, permitindo que o processo de irradiação das amostras não fosse significativo sobre a umidade das sementes.

Na Tabela 1 encontra-se o resumo da análise de regressão na análise de variância, representado pelos quadrados médios da germinação e do vigor das sementes de amendoim irradiadas com uma fonte de ${ }^{60} \mathrm{Co}$.

Os resultados das avaliações de vigor e germinação foram significativos para regressão linear e quadrática com o coeficiente de determinação $\left(\mathrm{R}^{2}\right)$ superior a $90 \%$. Pela Tabela 1 não houve efeito significativo para as demais regressões testadas ( $3^{\circ}$ e $4^{\circ}$ graus). Os tratamentos 12,0 e 24,0 kGy não foram incluídos nesta análise, em virtude de terem fornecido resultado zero, isto é, as sementes de amendoim não germinaram a partir da dose de $12 \mathrm{kGy}$. Os resultados da média e
Tabela 1. Resumo da análise de regressão para germinação e vigor das amostras de amendoim BRS Havana, irradiadas com uma fonte de ${ }^{60} \mathrm{Co}$

\begin{tabular}{ccccccc}
\hline \multirow{2}{*}{ F.V. } & \multirow{2}{*}{ G.L. } & \multicolumn{2}{c}{ Quadrados Médios } & \multicolumn{2}{c}{ Coeficiente Determinação $\left(\mathbf{R}^{2}\right)$} \\
Regressão Linear & 1 & $11356,90^{* *}$ & $11222,5^{* *}$ & 0,952145 & 0,961319 \\
Regressão Quadrática & 1 & $700,07^{*}$ & $617,78^{*}$ & 0,977974 & 0,985745 \\
Regressão Cúbica & 1 & $240,10 \mathrm{~ns}$ & $160,00 \mathrm{~ns}$ & - & - \\
Regressão 40 grau & 1 & $4,13 \mathrm{~ns}$ & $6,91 \mathrm{~ns}$ & - & - \\
Tratamentos & 4 & 3075,30 & $3001,80^{* *}$ & - & - \\
Resíduo & 15 & 87,80 & 93,87 & - & - \\
C.V. (\%) & & 23,02 & 19,45 & - & - \\
\hline
\end{tabular}

** Significativo a $1 \%$ de probabilidade pelo teste F; * significativo a $5 \%$ de probabilidade pelo teste $\mathrm{F}$; ns não significativo

da variância foram iguais a zero. Portanto, houve influência direta na homogeneidade das variâncias dos tratamentos. Neste caso, recomenda-se a omissão da análise de variância (Cochran, 1947).

Objetivando-se estimar a viabilidade (germinação e vigor) das diferentes doses de irradiação, selecionou-se a equação de regressão que melhor representa as doses, compreendendo a significância do teste $\mathrm{F}$ e o coeficiente de determinação $\left(R^{2}\right)$. A representação gráfica de cada variável com a respectiva equação se observa na Figuras $1 \mathrm{~A}$ e $1 \mathrm{~B}$.

Os resultados transcritos nas Figuras $1 \mathrm{~A}$ e $1 \mathrm{~B}$ tornam evidente o efeito das doses de irradiação gama sobre a viabilidade das sementes de amendoim, evidenciando efeito negativo, uma vez que, à medida em que se elevou a dose de radiação, diminui-se a viabilidade das sementes obtida pela
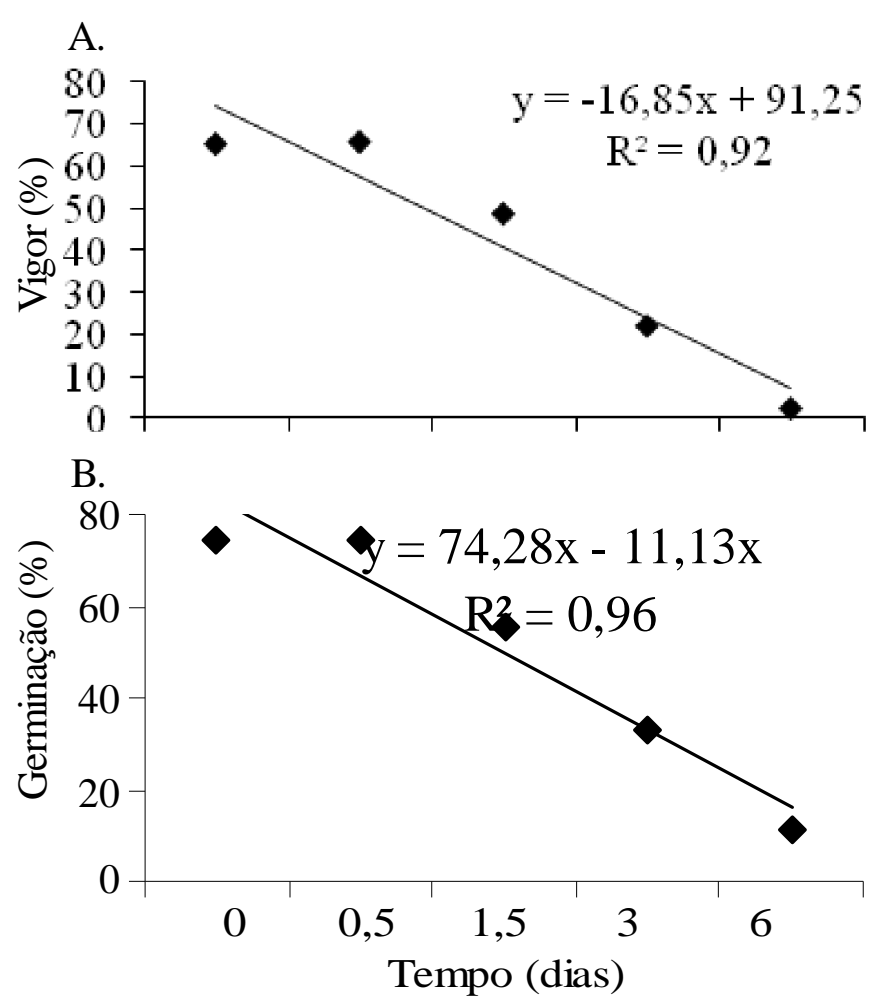

Figura 1. Representação gráfica da porcentagem da $1^{\mathrm{a}}$ contagem de germinação (vigor) (A) e germinação (B) $m$ relação às diferentes doses de radiação 
porcentagem de germinação e pela sua primeira contagem (vigor).

Conforme as informações dessas figuras, as porcentagens de germinação e de vigor das sementes não radiadas foram 74,5 e 65\%, respectivamente, reduzindo a viabilidade com o aumento da dose de radiação. A perda da germinação e do vigor se deve à radiossensibilidade do eixo embrionário. A diferença entre os resultados dos testes foi provocada pelo rigor do teste de vigor em relação ao de germinação.

Observa-se, ainda, mediante os resultados desses gráficos, uma forte diminuição da viabilidade das sementes irradiadas a partir da dose de 3,0 kGy, em que a percentagem do vigor obteve redução em $66 \%$ e a da germinação, em 55,7\%, em relação à dose de $0 \mathrm{kGy}$ (testemunha).

As equações de regressão das Figuras 1Ae 1B são importantes em razão de poderem informar o comportamento dos pontos intermediários entre os obtidos experimentalmente, relativos às doses de 0 a 3,0 kGy. Estudos sobre o tema concluíram que baixas doses de radiação possuem a capacidade de estimular os biossistemas, invertendo o efeito com o aumento da dose (Luckey, 1980). Esta teoria, denominada Hormese, ainda não é muito bem compreendida e é bastante questionada; isto poderia explicar a mesma porcentagem de germinação e vigor na testemunha com redução desses valores de germinação e vigor em função do aumento da dose de radiação.

Os referidos resultados são parcialmente concordantes com o princípio de Alara de proteção radiológica (ICRP, 1990), que considera prejudicial qualquer dose de radiação aos organismos. O mesmo autor relata que, com soja, trigo e milho, se obtiveram bons resultados de produtividade com as sementes desses produtos irradiadas, usando-se baixas doses antes da semeadura.

Tornou-se claro, neste estudo, que doses acima de 3,0 kGy, são altamente prejudiciais à viabilidade das sementes de amendoim. O resultado deste experimento está de acordo com outras pesquisas, envolvendo a germinação de sementes de outras espécies, como as de milho, soja e girassol, estudadas por Crede et al. (2004), Fanaro et al. (2004), Barros \& Arthur (2005). Em ambos os experimentos, o uso da radiação foi prejudicial à germinação.

\section{Micoflora}

De acordo com os exames realizados sob microscópio estereoscópico, os fungos que se desenvolveram sobre as sementes de amendoim irradiadas pertencem as espécies Aspergillus flavus, Aspergillus glaucus, Aspergilus niger e Penicillium spp. (Figuras 2A, 2B, 2C, 2D).

A porcentagem de contaminação por $A$. flavus diminui com o aumento da dose de irradiação (Figura 2A). Este fungo não foi encontrado nas doses de 2,5;3,0; 15,0 e 21,0 kGy e nas doses de 6,$0 ; 9,0 ; 12,0 ; 18,0$ e 24,0 kGy, a contaminação foi menor que $1 \%$; já a contaminação por A. glaucus foi maior nas doses de 0,5 e $15 \mathrm{kGy}, 10 \%$ (Figura 2B). Nas amostras que receberam 1,$5 ; 2,0 ; 2,5 ; 3,0 ; 6,0 ; 9,0 ; 12,0$ e 18,0 kGy a percentagem de contaminação foi menor que $3,5 \%$, enquanto a contaminação por A. niger diminuiu com o aumento da dose
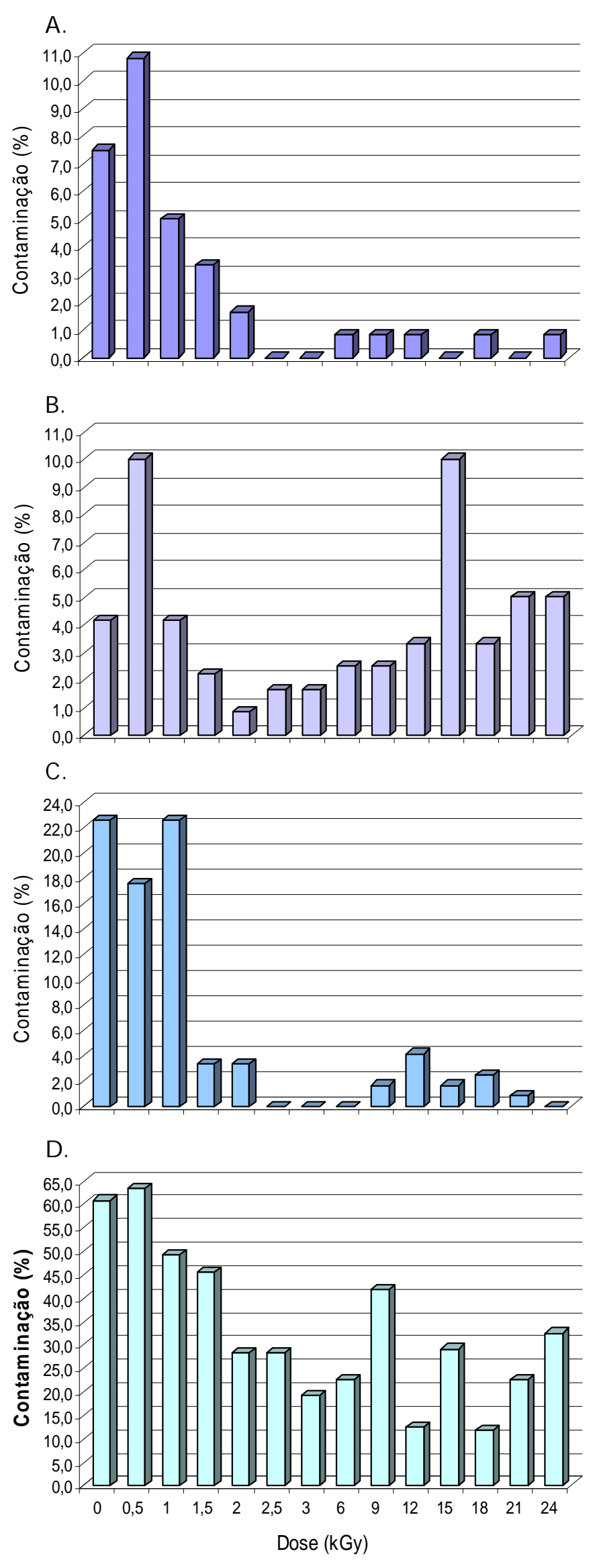

Figura 2. Representação gráfica da porcentagem de A. flavus (A), A.glaucus (B), A. niger $(C)$ e Penicilium spp. (D) em diferentes doses de radiação 
de irradiação aplicada (Figura 2C). As doses 0; 0,5 e 1,0 kGy apresentaram a maior contaminação, em média $20 \%$. Por outro lado, a contaminação por Penicillium spp foi diferente da observada para os demais fungos identificados (Figura 2D). Na testemunha (sem radiação), 60\% das sementes estavam contaminadas por Penicillium spp. Sempre que a dose de radiação foi aumentando, a percentagem de contaminação foi diminuindo, chegando a $32,5 \%$ na dose $24 \mathrm{kGy}$, ou seja, não foi possível eliminar este fungo nem com a maior dosagem de radiação utilizada.

Prado et al. (2006), testaram o efeito de diferentes níveis de radiação sobre a porcentagem fúngica de amendoim in natura, com e sem o processo de desinfecção dos grãos, em função do tempo de armazenamento e observaram que a radiação gama em doses acima de $10 \mathrm{kGy}$, foi capaz de eliminar a contaminação fúngica do amendoim em grãos armazenados durante 6 meses em temperatura ambiente, independente do processo de desinfecção; no entanto, mais estudos são necessários para se encontrar um equilíbrio entre as doses eficazes para o controle da microbiota fúngica e a utilização dessas sementes para germinação.

\section{CONCLUSÕES}

1. O tratamento com radiação gama afeta negativamente o vigor, a germinação das sementes de amendoim e a contaminação por fungos em sementes de amendoim.

2. As doses de 0,5 e 1,5 reduzem a germinação e o vigor acima de $50 \%$.

3. A dose de 3 kGy inviabiliza a utilização das sementes para o plantio.

4. Doses acima de $12 \mathrm{kGy}$ comprometem totalmente o vigor e a germinação das sementes.

5. Os fungos Aspergillus flavus, Aspergillus niger foram eliminados a partir da dose 2,0 kGy e o Aspergillus glaucus foi eliminado a partir da dose 3,0 kGy; entretanto Penicilium spp. permaneceu em mais de $30 \%$ das sementes em todos os tratamentos com radiação, não sendo eliminado nem com a dose de 24 kGy.

\section{LITERATURA CITADA}

Barros, A. C. de; Arthur, V. Determinação experimental da dose de redução do crescimento $\left(\mathrm{GR}_{50}\right)$ e da dose letal $\left(\mathrm{LD}_{50}\right)$ de soja irradiada por raios gama. Arquivo do Instituto de Biologia, v.72, n.2, p.249-253, 2005.

Brasil. Ministério da Agricultura. Regras para análise de sementes. Brasília: SNDA/LANARV. 1992. 188p.
Brock, R. D.; Franklin, I. R. The effect of desiccation, storage and radiation intensity on mutation rate in tomato pollen. Radiation Botany, v.6, p.171-179, 1966.

Bump, E. A.; YU, N. Y.; Brown, J. M. Radiosensitization of hypoxic tumor cells by depletion of intracellular glutathione. Science, v.217, p.544-545, 1982.

Cochran, W. G. Some consequences when the assumptions for the analysis of variance are not satisfied. Biometrics, v.3, p.2238, 1947.

CONAB - Companhia Nacional de Abastecimento. Previsão de safra 2007/2008. São Paulo. Ministério da Agricultura, Pecuária e Abastecimento. http://www.conab.gov.br/conabweb/download/safra/11 graos. 19 Ago. 2008.

Conger, B. V.; Carabia, J. V. Modification of the effectiveness of fission neutrons versus ${ }^{60} \mathrm{Co}$ gamma radiation in barley seeds by oxygen and seed water content. Radiation Botany, v.12, p.411-420, 1972.

Crede, R. G.; Baldasso, J. G.; Claudio, T. B.; Fanaro, G. B.; Guedes, R. L.; Sabundjian, I. T.; Vilavicêncio, A. L. C. H. Germinometria de grãos de milho (Zea mays) e soja (Glycine max) tratados por radiação ionizante. Arquivo Instituto de Biologia, v.71 (supl.), p.189-191. 2004.

Fanaro, G. B.; Baldasso, J. G.; Crede, R. G.; Claudio, T. B.; Sabundjian, I. T.; Guedes, R. L.; Villavicencio, A. L. C. H. Teste de germinação em sementes de girassol (Helianthus annus L.) irradiadas. Arquivo do Instituto de Biologia, v.71, p.178180. 2004.

ICRP - International Commission on Radiological Protection. Princípio da otimização, Oxford: Pergamon Press, 1990. 201p.

Luckey, T. D. Hormesis with ionizing radiation. Flórida: CRC Press, 1980. 4p.

Prado, G..; Carvalho, E. P de; Madeira, J. E. G. C.; Morais, V. A. D.; Oliveira, M. S.; Corrêa, R. F.; Cardoso, V. N. Efeito da irradiação gama $\left({ }^{60} \mathrm{Co}\right)$ na freqüência fúngica de amendoim in natura em função do tempo de prateleira. Ciência e Agrotecnologia, v.30, n.5, p.930-93. 2006.

Sanders, T. H.; Hill, R. A.; Cole, R. J.; Blankenship, P. D. Effect of drought on occurrenceof Aspergillus flavus in maturing peanuts. Journal of the American Oil Chemistry Society, v.58, p.966-970, 1981.

Santos, J. W. dos; Almeida, F. de A. C.; Beltrão, N. E. de M. ; Cavalcanti, F. B. Estatística experimental aplicada. 2.ed. Campina Grande: Embrapa Algodão/UFCG. 2007.

Santos, M. V. F. D. L. Resposta à radiação gama em sementes de milho (Zea mays L.) sob a influência de agentes físicos e químicos. UFV: Viçosa, 1993. 131p. Dissertação Mestrado

SAS/STAT User's Guide. In: SAS Institute. SAS Onlindoc: Version 8.2, cary: 2000. CD-Rom

Vieira, R. D.; Carvalho, N. M. Teste de vigor em sementes. Jaboticabal: FUMEP, 1994. 164p. 